\title{
Ankle Joint in Active Rheumatoid Arthritis
}

\section{Doaa A. Mohammed ${ }^{1}$, Zeinab M. Mahmoud ${ }^{2}$, Ahmed M Elsaman ${ }^{1}$}

\author{
1 Rheumatology and Rehabilitation Department, Faculty of Medicine, Sohag \\ University, Egypt \\ 2 Clinical Pathology Department, Faculty of Medicine, Sohag University, Egypt
}

\begin{abstract}
Background: Ultrasonography (US) is an imaging technique that proved to be useful for the assessment of articular and periarticular structures in different rheumatic diseases. The use of the US for ankle and foot usually requires a linear high-frequency transducer; a 7.5 to $15 \mathrm{MHz}$ probe is commonly used. This study aimed to evaluate ankle joint affection in active Rheumatoid arthritis and its value in the assessment of disease activity by using the US, and its correlation to disease duration, sex, and age.
\end{abstract}

Methods: A total of 63 patients with Rheumatoid arthritis according to the 2010 ACR/EULAR classification criteria. 20 age and sex-matched control volunteers were included. Evaluation of disease activity by DAS-28. US examination of both ankles was done for patients and controls. High-frequency US with a $12 \mathrm{MHZ}$ linear probe was used.

Results: Our results show that ankle synovitis was found significantly higher among RA patients $(18.3 \%)$ compared to controls $(2.5 \%)$; p-value $=0.013$. although tenosynovitis and erosions were higher among cases $(27 \%$ and $8.7 \%$; respectively) than controls (15\% and $0 \%$; respectively); the differences were non-significant. We found that ankle affection as seen by the US was significantly related to the activity of RA. Patients with higher DAS-28 scores were more like to have ankle affection and also to have bilateral ankle affection; both with significant differences.

Conclusion: It can be stated that ankle evaluation by the US should be considered more in RA assessment, and further studies should be done to uncover the underestimation of ankle involvement in RA patients.

\section{Introduction}

Ultrasonography (US) is an important and easy imaging technique proved to be helpful for the assessment of both articular and periarticular structures in all rheumatic diseases especially rheumatoid arthritis (RA) ${ }^{(1,2)}$. It provides much information about the musculoskeletal system such as synovitis, joint effusion, tenosynovitis, tendinitis and tendinopathy, tendons tears, bursitis, and erosions ${ }^{(1)}$.

Although symptoms suggesting ankle and foot involvement are very frequent in RA patients (around 20\% of all RA cases); their involvement is usually underestimated due to more concentration on hand and wrist involvement. Also, it may be difficult to distinguish between articular and peri-articular involvement in this area. Nearly all of the RA activity scores ignored or limited the importance of the ankle joint in its assessment ${ }^{(2)}$.

As plain radiographs provide very little information about soft tissue involveement and MRI is costly and is not 
widely available; musculoskeletal ultrasound (MSUS) can be a good alternative with both accuracy and safety; with also relatively low-cost ${ }^{(2)}$.

Ankle and foot the US usually require a linear high-frequency transducer; with 7 to $15 \mathrm{MHz}$ probe commonly used. Colour Doppler (CD) and more commonly Power Doppler (PD) techniques provide useful additional information regarding the presence and extent of soft tissue hyperemia ${ }^{(I)}$.

Multiplanar, bilateral and dynamic assessments should always be performed when scanning either the foot or ankle.

\section{Aim of the work:}

To evaluate ankle joint affection in active RA and its value in assessment of disease activity by using the US, and its correlation to disease duration, sex, and age.

\section{Patients and Methods:}

Design: Prospective comparative clinical study.

\section{Patients:}

- A total of 63 patients with RA were candidates for our study. All the patients have been considered as having RA according to the 2010 ACR/EULAR classification criteria $^{(3)}$.

- 20 age and sex-matched control volunteers were enrolled in the study for comparison purposes.

\section{Inclusion criteria:}

- Patients having RA inactivity (DAS score of more than 2.6)

- With a disease duration of more than 6 months.

- Aged more than 18 years old.

\section{Exclusion criteria:}

- Patients having other autoimmune rheumatic diseases.

- Patients with a history of ankle disease or trauma.

- Patients with RA in remission (DAS<2.6).

\section{Methods: \\ Evaluation of disease activity by DAS-28}

Disease activity was evaluated for all patients using DAS-28 score, based on ESR, according to the following calculation: DAS28 $=0.56 *$ square root (tender joints) $+0.28 *$ square root $($ swollen joints $)+0.70 * \log (\mathrm{ESR})+$ 0.014 (global activity score) ${ }^{(4)}$.

ESR, CRP, and Rheumatoid factor was done for all patients.

US examination of both ankles was done for patients and controls. A highfrequency US machine with a $12 \mathrm{MHZ}$ linear probe (Siemens Corporation, Germany) was used. Both of the rheumatologist and the clinical pathologist was blinded for the nature of the patient.

\section{Ethical Considerations:}

- This research was revised by the Scientific Ethical Committee of Sohag

University Hospital.

- Informed written consent was taken from all patients after a detailed explanation

of the aim and steps of the study.

\section{Statistical analysis:}

- Statistical package for social sciences (IBM-SPSS), version 24 IBM- Chicago, USA (May 2016) was used for statistical data analysis.

- Data expressed as mean, standard deviation (SD), number, and percentage. Mean and the standard deviation was used as descriptive values for quantitative data, while numbers and percentages were used to describe qualitative data.

- A Student t-test was used to compare the means between two groups, and one-way analysis of variance (ANOVA) test was used to compare means of more than two groups. Mann Whitney test was 
used instead of Student t-test in case of non-parametric data.

- For all these tests, the level of significance (P-value) can be explained as:

$\circ$ No significance $\mathrm{P}>0.05$

$\circ$ Significance $\mathrm{P}<0.05$

$\circ$ High significance $\mathrm{P}<0.001$.

\section{Results}

Our study found that the cases and controls were age and sex-matched, with a non-significant difference between the two groups. Around two-thirds of both groups were females, and the mean age of them was 35 years, with a range from 20-50 years.

We found that both ESR and CRP were significantly higher among patients compared to controls. Also, we found that nearly $78 \%$ of cases had positive RF, compared to only $15 \%$ of the controls, of course with a highly significant difference.

We found that around half of the cases $(44.4 \%)$ had positive ankle US findings, compared to only $20 \%$ among the controls. However, both cases and controls showed predominantly affected right ankle compared to the left ankle. The difference here was nonsignificant. Our results show that ankle synovitis was found significantly higher among RA patients (18.3\%) compared to controls $(2.5 \%) ; \mathrm{p}$-value $=$ 0.013 . On the other hand, although tenosynovitis and erosions were higher among cases $(27 \%$ and $8.7 \%$; respectively) than controls (15\% and $0 \%$; respectively); the differences were non-significant, probably due to high ratio of tenosynovitis among controls and due to the limited number of cases with frank erosions (figure 1).

We found that the mean disease duration of RA patients in the study was around 2 years, with a range from 6 to 60 months. Regarding disease activity, most of the cases $(71 \%)$ had low disease activity, with $24 \%$ had intermediate disease activity and only less than $5 \%$ had high disease activity (Table 1).

We found that ankle affection as seen by the US was significantly related to the duration of RA. Patients with longer disease duration were more like to have ankle affection and also to have bilateral ankle affection. Regarding individual ankle findings, we found that the earliest finding was tenosynovitis, followed by synovitis, and lastly erosions. However, the difference in the mean disease duration was only significant between tenosynovitis and erosions.

We found that ankle affection as seen by the US was significantly related to the activity of RA. Patients with higher DAS-28 scores were more like to have ankle affection and also to have bilateral ankle affection; both with significant differences. Regarding individual ankle findings, we found that tenosynovitis was associated with the lowest DAS-28 score, followed by erosions, while synovitis had the highest DAS-28 score. However, the difference in the mean disease activity as regards individual US findings were all non-significant (figure 2).

\section{Discussion}

Over the last decade, the usage of musculoskeletal US in the diagnosis and follow-up of the disease activity in RA has improved dramatically. Many scoring systems have been developed and tested in different studies for this purpose using both GS and PDUS. However, the majority of these scores did not include the ankle and foot area (5).

Although Suzuki et al had assessed thoroughly the ankle joint and ankle area among RA patients; they fell into some weak points. These weak points included the non-inclusion of erosion evaluation, no correlation between ankle US findings with clinically 
assessed disease activity, lack of relationship between disease activity and disease duration at one side and bilateral ankle involvement on the other side, and lastly, their study was not blinded because it was a retrospective one ${ }^{(6) .}$

Naredo et al. assessed the ankle in their study, but they focused only on the anterior tibiotalar recess, anterior, medial and lateral tendon sheaths but they did not estimate erosions ${ }^{(7) .}$

In our study, tenosynovitis was found more frequently than synovitis. Suzuki et al. results were similar to ours ${ }^{(6)}$. On the contrary, the study of Alsuwaidi et $\boldsymbol{a l}$. found that synovitis more often than tenosynovitis ${ }^{(8 \& 9)}$. The fact that they included long-standing RA patients, with a median disease duration of 5 years and active RA with a median DAS28-ESR score of 5 might explain why their study gave different results than ours.

In our study, we found a positive relation was found between disease duration and the incidence of erosions.

Ohrndorf et al. in their study to evaluate erosion showed that there was a relation between erosions and both disease activity (measured by DAS28ESR score) and disease duration. However, they did not include the ankle in their study ${ }^{(10)}$. Other studies also showed a positive relationship between erosion and disease duration ${ }^{1}$ \& 11).

The liability for bilateral involvement of the ankles was found to be correlated with longer disease duration. There is significant controversy regarding symmetrical affection in RA patients. Some of these studies stated that the dominant side is more affected while others insisted on the hypothesis that RA is a largely symmetrical disease ${ }^{(12 \& 13)}$.

The earliest ankle finding found in our study was tenosynovitis, followed by synovitis; while erosions were the latest one. This was typically similar to the study of Suzuki et al. who found that tenosynovitis was recorded earlier than synovitis ${ }^{(6)}$.

Our study found also that the ankle region was much more largely involved among patients with higher disease activity as detected by DAS28ESR score. This was maximally significant regarding tenosynovitis, followed by synovitis, but not for erosions. This was somewhat different from Ohrndorf et al. who found that all of the synovitis, tenosynovitis and erosion were significantly related to disease activity (14). In the study of Gutierrez et al with disease duration and DAS-28 ESR score values comparable to our study, but they failed to demonstrate a significant relationship between disease activity and ankle involvement. This difference may be due to their exclusion of any cases with symptomatic ankles from the start and including only those with active RA but with "under-estimated" non-symptomatic ankle involvement (15)

It is important to state that our study excluded both Tendon Achilles and plantar fascia. We did not include them because this may lengthen the examination time and review of the literature showed the limited value of them in ankle evaluation. We believed that an examination of these structures may be more beneficial in patients with seronegative spondyloarthropathy
rather than $\mathrm{RA}^{(8 \& 9) .}$

\section{Conclusion:}

The ankle region is underestimated in the evaluation of RA patients, and its evaluation by MSUS should be more focused to uncover this underestimation. 


\section{Table and figure legend}

\begin{tabular}{llll}
\hline Item & & Value & \\
\cline { 1 - 2 } $\begin{array}{llll}\text { Disease duration in } \\
\text { months }\end{array}$ & Mean \pm SD & $22.68 \pm 9.63$ & \\
DAS-28 & Median(range) & $22(6-60)$ & Table 1. Clinical data \\
& Mean \pm SD & $3.05 \pm 0.66$ & \\
& Median(range) & $2.80(2.6-5.8)$ & of the study groups \\
& Low disease activity & $45(71.4 \%)$ & \\
& Intermediate disease activity & $15(23.8 \%)$ & \\
& High disease activity & $3(4.8 \%)$ & \\
\hline
\end{tabular}

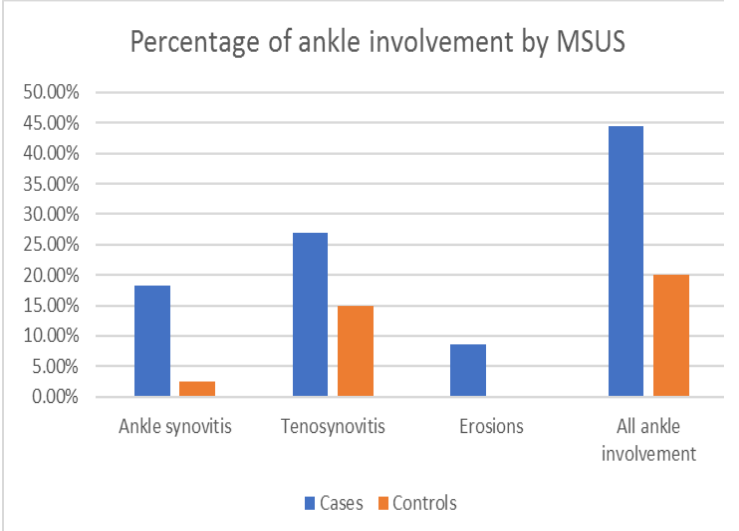

Figure 1: Ankle involvement in RA cases compared to controls

\section{References:}

1. Wakefield RJ, Gibbon WW, Conaghan PG et al. The value of sonography in the detection of bone erosions in patients with rheumatoid arthritis. Arthritis Rheum 2000; 43:2762-70.

2. Lopez-Ben $\mathbf{R}$, Bernreuter WK, Moreland $\mathbf{L W}$ et al. Ultrasound detection of bone erosions in rheumatoid arthritis: a comparison to routine radiographs of the hands and feet. Skeletal Radiology 2004; 33: 8084 .

3. Aletaha D, Neogi T, Silman AJ, et al. 2010 rheumatoid arthritis classification criteria: an American College of Rheumatology/ Euro-pean League Against Rheumatism collaborative initiative. Ann Rheum Dis. 2010; 69(9): 1580-1588.

4. Source: DAS-Score.nl. Available at http://www.das-score.nl/www.das-

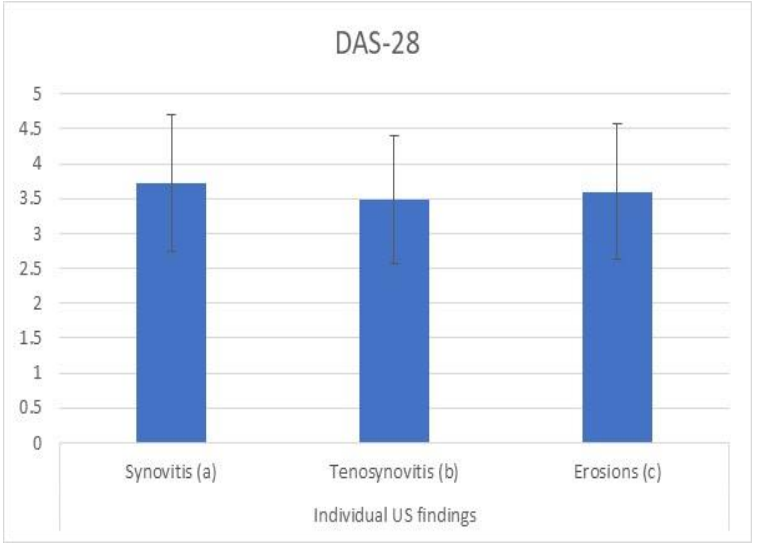

Figure 2: Comparison between DAS28 score and individual US findings among the study population

score.nl/index.html. Accessed February 5, 2009.

5. Naredo E, Valor L, De la Torre I et al. Ultrasound joint inflammation in rheumatoid arthritis in clinical remission: how many and which joints should be assessed? Arthritis Care \& Research 2013; 65:512-17

6. Suzuki T, Okamoto A. Ultrasound examination of symptomatic ankles in shorter-duration rheumatoid arthritis patients often reveals tenosynovitis. Clin Exp Rheumatol. 2013;31:281284 .

7. Naredo E, Rodriguez M, Campos C et al. Validity, reproducibility, and responsiveness of a twelve-joint simplified power Doppler ultrasonographic assessment of joint inflammation in rheumatoid arthritis. Arthritis Care \& Research 2008; 59:515-22. 
8. Alsuwaidi M, Ehrenstein B, Fleck M et al. Asymptomatic Versus Symptomatic Ankle Joints in Rheumatoid Arthritis: A HighResolution B-Mode and Power Doppler Ultrasound Study. Arthritis Care \& Research 2016; 68:861-64.

9. Alsuwaidi M, Ehrenstein BP, Fleck M et al. 2013 Ankle Joint Involvement In Rheumatoid Arthritis. An Ultrasound Study Using High Resolution-and Colour Doppler Ultrasound. Arthritis And Rheumatism: Wiley-Blackwell 111 River St, Hoboken 07030-5774, NJ USA, S839S39.

10.Ohrndorf S, Messerschmidt J, Reiche B et al. Evaluation of a new erosion score by musculoskeletal ultrasound in patients with rheumatoid arthritis. Clinical rheumatology 2014; 33:1255-62.

11.Schett G, Gravallese E. Bone erosion in rheumatoid arthritis: mechanisms, diagnosis, and treatment. Nature Reviews Rheumatology 2012; 8:65664.
12.Zangger P, Keystone EC, Bogoch ER. Asymmetry of small joint involvement in rheumatoid arthritis: prevalence and tendency towards symmetry over time. Joint Bone Spine 2005; 72:241-47.

13.Guillén Astete C, Boteanu A, Zea Mendoza A. Comparison of Prevalence of Synovitis by Ultrasound Assessment in Subjects Exposed or Not to Self-Reported Physical Overexertion: The Monday's Synovitis. The Scientific World Journal 2014; 2014.

14.Ohrndorf S, Glimm A-M, Burmester G-R et al. Musculoskeletal ultrasound scoring systems: assessing disease activity and therapeutic response in rheumatoid arthritis. International Journal of Clinical Rheumatology 2011;6:57-65.

15.Gutierrez M, Pineda C, Salaffi F et al. Is ankle involvement underestimated in rheumatoid arthritis? Results of a multicenter ultrasound study. Clinical rheumatology 2016; 35:2669-78. 